\title{
The degree/diameter problem in maximal planar bipartite graphs (Extended abstract)
}

\author{
C. Dalfó, C. Huemer ${ }^{1,3}$ \\ Dept. de Matemàtica Aplicada IV \\ Universitat Politècnica de Catalunya \\ Barcelona, Catalonia \\ J. Salas ${ }^{2,4}$ \\ IIIA-CSIC \\ Bellaterra (Barcelona), Catalonia
}

\begin{abstract}
The $(\Delta, D)$ (degree/diameter) problem consists of finding the largest possible number of vertices $n$ among all the graphs with maximum degree $\Delta$ and diameter $D$. We consider the $(\Delta, D)$ problem for maximal planar bipartite graphs, that are simple planar graphs in which every face is a quadrangle. We obtain that for the $(\Delta, 2)$ problem, the number of vertices is $n=\Delta+2$; and for the $(\Delta, 3)$ problem, $n=3 \Delta-1$ if $\Delta$ is odd and $n=3 \Delta-2$ if $\Delta$ is even. Then, we study the general case $(\Delta, D)$ and obtain that an upper bound on $n$ is approximately $3(2 D+1)(\Delta-2)^{\lfloor D / 2\rfloor}$ and another one is $C(\Delta-2)^{\lfloor D / 2\rfloor}$ if $\Delta \geq D$ and $C$ is a sufficiently large constant. Our upper bound improve for our kind of graphs the one given by Fellows, Hell and Seyffarth for general planar graphs. We also give a lower bound on $n$ for maximal planar bipartite graphs, which is approximately $(\Delta-2)^{k}$ if $D=2 k$, and $3(\Delta-3)^{k}$ if $D=2 k+1$, for $\Delta$ and $D$ sufficiently large in both cases.
\end{abstract}

Keywords: $(\Delta, D)$ problem, maximal planar bipartite graphs. 


\section{Introduction}

We consider simple graphs $G=G(V, E)$ that are bipartite, planar and with the maximum possible number of edges. In a bipartite graph, each cycle has an even length. If a graph can be drawn on the plane without any crossing of its edges, then the graph is called planar. A planar bipartite graph is maximal if when we add a new edge, the graph obtained is no longer planar or bipartite. A maximal planar bipartite graph divides the plane only into quadrangles (see Ringel [7]). From the Euler characteristic $|V|-|E|+|F|=2$, which relates the numbers of vertices $n=|V|$, edges $|E|$ and faces $|F|$ in a planar embedding of $G$, and the fact that each face is incident to four edges, one obtains the well-known relations $|E|=2 n-4$ and $|F|=n-2$.

The $(\Delta, D)$ problem consists of finding the maximum possible number of vertices $n=|V|$ in a graph $G$ with maximum degree $\Delta$ and diameter $D$. This is a prominent topic in graph theory, with results obtained for many cases. Information about this problem for graphs in general can be found in the comprehensive survey by Miller and Šráň [6] and for planar graphs also on the web page by Loz, Pérez-Rosés, and Pineda-Villavicencio [5].

We study the $(\Delta, D)$ problem for maximal planar bipartite graphs. In this text, we omit most of the proofs because of lack of space. We show in Section 2 , that in the $(\Delta, 2)$ problem $n=\Delta+2$ and that only the complete bipartite graph $K_{2, \Delta}$ satisfies this equation. Moreover, we solve the $(\Delta, 3)$ problem and prove that $n=3 \Delta-1$ if $\Delta$ is odd, and $n=3 \Delta-2$ if $\Delta$ is even. In Section 3, we study the general case $(\Delta, D)$ and obtain that $n$ is bounded from above by approximately $3(2 D+1)(\Delta-2)^{\lfloor D / 2\rfloor}$. For the case $\Delta \geq D$, we also obtain the upper bound $n \leq C(\Delta-2)^{\lfloor D / 2\rfloor}$, for some constant $C$. Our upper bound improve for our kind of graphs the one given by Fellows, Hell and Seyffarth for general planar graphs. We also give a lower bound on $n$ for maximal planar bipartite graphs, which is approximately $(\Delta-2)^{k}$ if $D=2 k$, and $3(\Delta-3)^{k}$ if $D=2 k+1$, for $\Delta$ and $D$ sufficiently large in both cases. The

\footnotetext{
${ }^{1}$ Research of C. Dalfó supported by the Ministry of Education and Science, Spain, and the European Regional Development Fund under project MTM2011-28800-C02-01, and by the Catalan Research Council under project 2009SGR1387. Research of C. Huemer partially supported by projects MEC MTM2012-30951 and Gen. Cat. DGR 2009SGR1040, 2014SGR46 and ESF EUROCORES programme EuroGIGA, CRP ComPoSe: grant EUIEURC-2011-4306.

2 Research supported by the Ministry of Education and Science, Spain, and the European Regional Development Fund (ERDF) under project MTM2011-28800-C02-02.

3 Email: \{cristina.dalfo, clemens. huemer\} @upc.edu

4 Email: julian.salas@iiia.csic.es
} 
precise bounds are given in that section.

\section{The $(\Delta, 2)$ and $(\Delta, 3)$ problems in maximal planar bi- partite graphs}

For maximal planar bipartite graphs with diameter $D=2$, we solve the $(\Delta, 2)$ problem with the following result.

Proposition 2.1 Consider a maximal planar bipartite graph $G$ with diameter $D=2$, maximum degree $\Delta$ and maximum number of vertices $n$, then $n=$ $\Delta+2$. The only graph that satisfies this equation is the complete bipartite graph $K_{2, \Delta}$.

For maximal planar bipartite graphs with diameter $D=3$, our main result is the following.

Theorem 2.2 Consider a maximal planar bipartite graph $G$ with diameter $D=3$, maximum degree $\Delta$ and maximum number of vertices $n$, then

$$
n=\left\{\begin{array}{l}
3 \Delta-1 \text { if } \Delta \text { is odd } \\
3 \Delta-2 \text { if } \Delta \text { is even } .
\end{array}\right.
$$

\section{The $(\Delta, D)$ problem in maximal planar bipartite graphs}

\subsection{An upper bound}

Fellows, Hell and Seyffarth [2] obtained bounds on the $(\Delta, D)$ problem for planar graphs applying the following theorem by Lipton and Tarjan [4].

Theorem 3.1 ([4]) Let $G$ be a planar graph on $n$ vertices containing a spanning tree of radius $r$. Then $V(G)$ can be partitioned into sets $A, B$ and $C$ such that no edges join vertices in $A$ with vertices in $B,|A| \leq \frac{2}{3} n,|B| \leq \frac{2}{3} n$, and $|C| \leq 2 r+1$.

Clearly, this theorem also holds for maximal planar bipartite graphs. We give an upper bound on the number of vertices for this kind of graphs. The cases $D=2$ and $D=3$ are studied in Section 2. No maximal planar bipartite graphs with $\Delta=3$ has more than $n=8$ vertices. Therefore, we assume that $D \geq 4$ and $\Delta \geq 4$.

Our aim is to give an upper bound on $n$, computing from each vertex of $C$ the maximum possible number of vertices at distance at most $\lfloor D / 2\rfloor$. We 


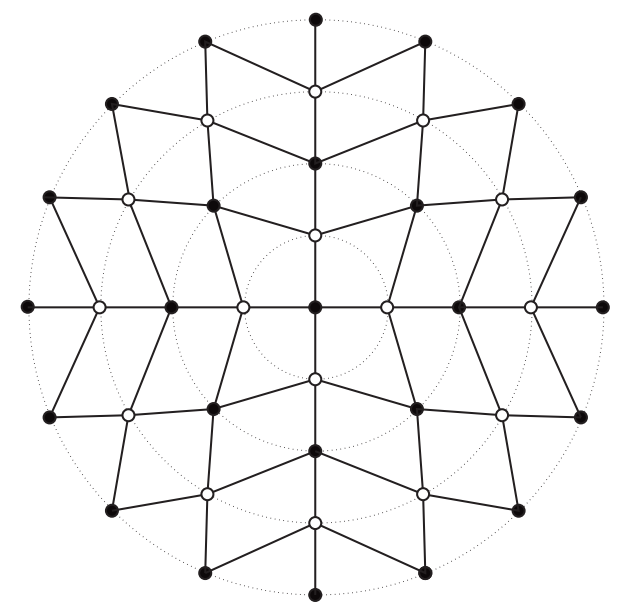

Figure 1. An almost maximal subgraph for $\Delta=4$.

build a subgraph adding vertices at distance $i$ from a given (root) vertex of $C$ in step $i(0 \leq i \leq\lfloor D / 2\rfloor)$, to obtain a planar bipartite graph (which is almost maximal, meaning that all its interior faces are quadrangles), as shown in Fig. 1. This is done in a similar way to the tree built to find the Moore bound (see Miller and Širáň [6]).

Let $n_{i}$ be the number of vertices at distance $i$ (for $0 \leq i \leq\lfloor D / 2\rfloor$ ). For the case $\Delta>4$ and $D \geq 4, n_{i}$ follows the recurrence

$$
n_{i}=(\Delta-2) n_{i-1}-n_{i-2}
$$

with $i \geq 3$. Solving this recurrence equation with a generating function, we obtain

$$
n_{i}=\frac{\Delta}{\sqrt{\Delta(\Delta-4)}}\left[\left(\frac{\Delta-2+\sqrt{\Delta(\Delta-4)}}{2}\right)^{i}-\left(\frac{\Delta-2-\sqrt{\Delta(\Delta-4)}}{2}\right)^{i}\right]
$$

Thus, the total number of vertices $n=\sum_{i=0}^{\lfloor D / 2\rfloor} n_{i}$ is obtained as the difference of two geometric series. With this result, and with a similar approach as in Fellows, Hell, and Seyffarth [2], that is using Theorem 3.1, we obtain the following theorem.

Theorem 3.2 Let $G$ be a maximal planar bipartite graph on $n$ vertices with maximum degree $\Delta \geq 4$ and diameter $D \geq 4$. Then, 


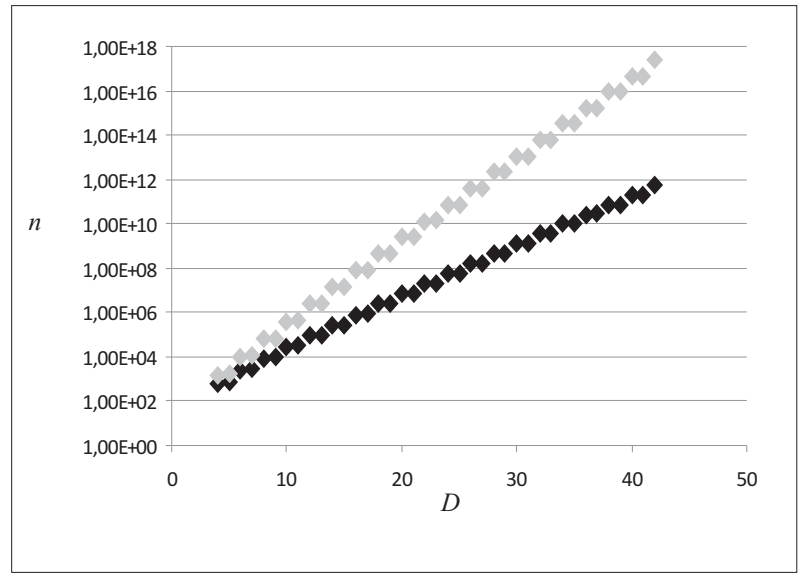

Figure 2. Plot of the $\log$ (base 10) of the number of vertices $n$ with respect to the diameter $D$ according to our bound given by Eq. (1) (black points) and the one by Fellows, Hell and Seyffarth given by Eq. (2) (grey points), for $\Delta=5$ and $4 \leq D \leq 42$.

(a) If $\Delta=4: n \leq 6(2 D+1)\left(\left\lfloor\frac{D}{2}\right\rfloor^{2}+\left\lfloor\frac{D}{2}\right\rfloor+1\right)$.

(b) If $\Delta>4$ :

$$
\begin{aligned}
n \leq & 3(2 D+1)\left[\frac { \sqrt { \Delta ( \Delta - 4 ) } } { 2 ( \Delta - 4 ) ^ { 2 } } \left[(\Delta-4+\sqrt{\Delta(\Delta-4)})\left(\frac{\Delta-2-\sqrt{\Delta(\Delta-4)}}{2}\right)^{\lfloor D / 2\rfloor+1}\right.\right. \\
& -2 \sqrt{\Delta(\Delta-4)} \\
& \left.\left.+(4-\Delta+\sqrt{\Delta(\Delta-4)})\left(\frac{\Delta-2+\sqrt{\Delta(\Delta-4)}}{2}\right)^{\lfloor D / 2\rfloor+1}\right]+2\right]
\end{aligned}
$$

which is approximately $3(2 D+1)\left[(\Delta-2)^{\lfloor D / 2\rfloor}+1\right]$ if $\Delta$ is sufficiently large.

The upper bound given by Fellows, Hell and Seyffarth [2] for planar graphs is

$$
n \leq 3(2 D+1)\left(2 \Delta^{\lfloor D / 2\rfloor}+1\right) .
$$

As our graphs are planar, this bound also applies to maximal planar bipartite graphs, but our bound is much better for this kind of graphs. See an example for $\Delta=5$ in Fig. 2, with the values of our bound given by Theorem 3.2 and the one by Fellows, Hell and Seyffarth.

We also give an alternative upper bound for the $(\Delta, D)$ problem of the form $n<C(\Delta-2)^{\lceil D / 2\rceil}$, for some constant $C$, which improves the bound of Theorem 3.2 by a factor $D$, when $D$ is even and sufficiently large. However, it remains for further research to determine the smallest value of $C$ for which 
this bound holds. It is based on the following theorem of Chepoi, Estellon, and Vaxès [1]. The ball of center $v \in G$ and radius $k$ consists of all vertices of $G$ at distance at most $k$ from $v$.

Theorem 3.3 ([1]) There exists a constant $C$ such that any planar graph $G$ of diameter $D \leq 2 k$ can be covered with at most $C$ balls of radius $k$.

As for a lower bound for Theorem 3.3, Gavoille, Peleg, Raspaud, and Sopena in [3] presented a family of planar graphs which requires $C \geq 4$.

Corollary 3.4 There exists a constant $C$ such that each maximal planar bipartite graph $G$ with maximum degree $\Delta$ and diameter $D$ has at most $n \leq$ $C(\Delta-2)^{\lceil D / 2\rceil}$ vertices.

We further strengthen the bound for the $(\Delta, D)$ problem given in Corollary 3.4 to $C(\Delta-2)^{\lfloor D / 2\rfloor}$, for the case $D$ odd and $\Delta \geq D$. We use the $N$-separator theorem by Tishchenko $[8,9]$ to obtain the following result.

Theorem 3.5 There exists a constant $C$ such that each maximal planar bipartite graph $G$ with maximum degree $\Delta$ and diameter $D$, for $\Delta \geq D$, has at most $n \leq C(\Delta-2)^{\lfloor D / 2\rfloor}$ vertices.

\subsection{A lower bound}

In this section we present maximal planar bipartite graphs $G_{\Delta, D}$, with given maximum degree $\Delta$ and diameter $D$, which have a large number $n=n\left(G_{\Delta, D}\right)$ of vertices.

The graph which attains the bound of Theorem $3.6(a)$ is based on the one depicted in Fig. 1; from a given root vertex we build a planar bipartite graph adding the maximum number of vertices at distance $i$ from the root in step $i$ (for $0 \leq i \leq D / 2$ ). We draw this graph on a sphere, with the root placed on the north pole and the vertices at distance $D / 2$ from the root are placed on the equator. Then we add a copy of this graph on the lower hemisphere, with the root on the south pole, and such that the vertices at distance $D / 2$ from the south pole are identified with those already placed on the equator. For the bounds $(b)$ and $(c)$ of Theorem 3.6, we use an iterative construction: We start with the graph on the left of Fig. 3 and then we substitute the dotted edges by complete bipartite subgraphs $K_{2, t}$ (where the value of $t$ differs according to the subcases of Theorem 3.6). The generic step of this construction is shown on the right of Fig 3. In each step of this construction, the diameter of the graph increases by 2 units. 
Theorem 3.6 $a)$ For any diameter $D=2 k(k \geq 1)$ and maximum degree $\Delta$ $(\Delta \geq 5)$, there exists a maximal planar bipartite graph $G_{\Delta, D}$ whose number of vertices $n\left(G_{\Delta, D}\right)$ is

$$
\frac{\Delta(\Delta-2+\sqrt{\Delta(\Delta-4)})^{k}+\Delta(\Delta-2-\sqrt{\Delta(\Delta-4)})^{k}}{(\Delta-4) 2^{k}}-\frac{8}{\Delta-4},
$$

which is approximately $(\Delta-2)^{k}$, for $\Delta$ and $D$ sufficiently large.

(b) For any diameter $D=2 k+1(k \geq 1)$ and odd maximum degree $\Delta(\Delta \geq 9)$, there exists a maximal planar bipartite graph $G_{\Delta, D}$ whose number of vertices $n\left(G_{\Delta, D}\right)$ is

$$
\begin{array}{ll}
n\left(G_{\Delta, 3}\right)=3 \Delta-1 & \text { for } D=3, \\
n\left(G_{\Delta, 5}\right)=3 \Delta^{2}-21 \Delta+26 & \text { for } D=5, \\
n\left(G_{\Delta, 2 k+1}\right)=3 \Delta^{2}-21 \Delta+26+\frac{3(\Delta-7)(\Delta-2)^{2}\left((\Delta-3)^{k-2}-1\right)}{(\Delta-4)} & \text { for } D=2 k+1 \\
& \text { and } k>2,
\end{array}
$$

which is approximately $3(\Delta-3)^{k}$, for $\Delta$ and $D$ sufficiently large.

(c) For any diameter $D=2 k+1(k \geq 1)$ and even maximum degree $\Delta(\Delta \geq$ $10)$, there exists a maximal planar bipartite graph $G_{\Delta, D}$ whose number of vertices $n\left(G_{\Delta, D}\right)$ is

$$
\begin{array}{ll}
n\left(G_{\Delta, 3}\right)=3 \Delta-2 & \text { for } D=3, \\
n\left(G_{\Delta, 5}\right)=3 \Delta^{2}-22 \Delta+26 & \text { for } D=5, \\
n\left(G_{\Delta, 2 k+1}\right)=3 \Delta^{2}-22 \Delta+26+\frac{(3 \Delta-22)(\Delta-2)^{2}\left((\Delta-3)^{k-2}-1\right)}{(\Delta-4)} & \text { for } D=2 k+1 \\
& \text { and } k>2,
\end{array}
$$

which is approximately $3(\Delta-3)^{k}$, for $\Delta$ and $D$ sufficiently large.

\section{References}

[1] Chepoi, V., B. Estellon, and Y. Vaxès, On covering planar graphs with a fixed number of balls, Discrete Comput. Geom. 37 (2007) 237-244.

[2] Fellows, M., P. Hell, and K. Seyffarth, Large planar graphs with given diameter and maximum degree, Discrete Appl. Math. 61 (1995) 133-153. 

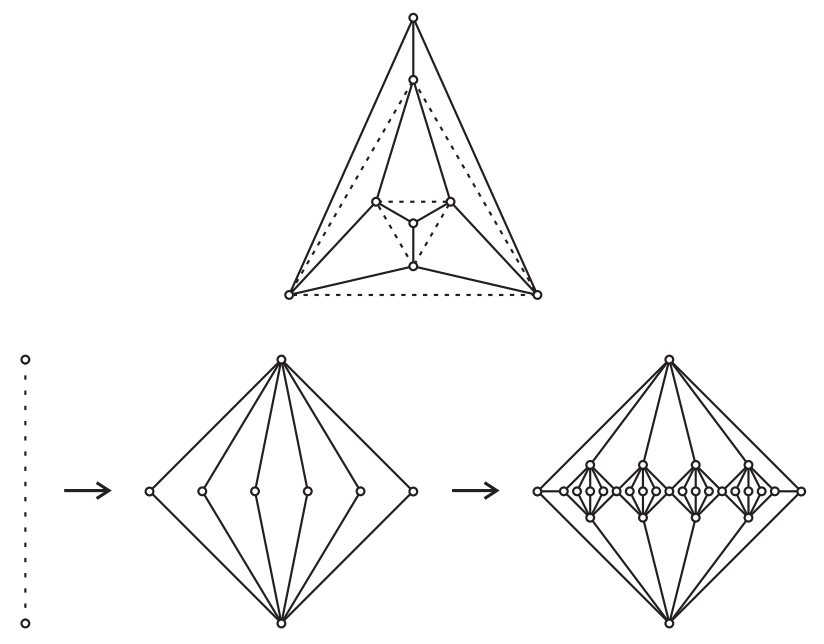

Figure 3. The iterative construction.

[3] Gavoille, C., D. Peleg, A. Raspaud, and E. Sopena, Small k-dominating sets in planar graphs with applications, "27th International Workshop on GraphTheoretic Concepts in Computer Science 2001", Lect. Notes Comput. Sc. 2204 (2001) 201-216, Springer-Verlag, Berlin.

[4] Lipton, R.J., and R.E. Tarjan, A separator theorem for planar graphs, SIAM J. Appl. Math. 36 (1979) 177-189.

[5] Loz, E., H. Pérez-Rosés, and G. Pineda-Villavicencio, The degree/diameter problem for planar graphs, http://combinatoricswiki.org/wiki, 2008.

[6] Miller, M., and J. Širáň, Moore graphs and beyond: A survey of the degree/diameter problem, Electron. J. Combin. 20(2) (2013) \#DS14v2.

[7] Ringel, G., Two trees in maximal planar bipartite graphs, J. Graph Theory $\mathbf{1 7}$ (1993) 755-758.

[8] Tishchenko, S.A., Maximum size of a planar graph with given degree and even diameter, Eur. J. Combin. 33 (2012) 380-396.

[9] Tishchenko, S.A., N-separators in planar graphs, Eur. J. Combin. 33 (2012) 397-407. 